\title{
Flat-Coated Retriever
}

National Cancer Institute

\section{Source}

National Cancer Institute. Flat-Coated Retriever. NCI Thesaurus. Code C53871.

The Flat-Coated Retriever is a black or liver-colored retriever with a sleek, medium-length, thick coat with well-feathered legs, tail and chest. The moderately small ears lay flat against the head. The chest is deep and wide. Height: 22-23 inches $(56-58 \mathrm{~cm}$.) Weight: 60-70 pounds (27-32 kg.) 